\title{
Pairing correlations with the Gogny force
}

\author{
N. Pillet ${ }^{1, a}$, J.-F. Berger ${ }^{1}$, E. Caurier ${ }^{2}$, H. Goutte ${ }^{1}$, N. Sandulescu ${ }^{3}$, and P. Schuck ${ }^{4}$ \\ 1 DPTA/Service de Physique nucléaire, CEA/DAM Île-de-France, BP. 12, 91680 Bruyères-le-Châtel, France \\ 2 Département Recherches Subatomiques, Institut Pluridisciplinaire Hubert Curien, 23 rue du Loess, BP. 28, 67037 Strasbourg, France \\ 3 Institute of Physics and Nuclear Engineering, 76900 Bucharest, Romania \\ ${ }^{4}$ Institut de Physique Nucléaire, CNRS UMR 8608, 91406 Orsay, France
}

\begin{abstract}
In this contribution we present two separated studies that deal with pairing correlations. The first one corresponds to a new development that aims to include correlations beyond mean field in an unified way and which is called Variational multiparticle-multihole (mpmh) configuration mixing method. Its main characteristic is that it conserves explicitly particle numbers unlike the usual HFB or BCS approaches. A comparison with BCS calculations shows that it is able to restore many correlations in weak pairing regime. The second study is devoted to the localisation and size of Cooper pairs in nuclei. We have defined the Cooper pair wave function in HFB approach. The main feature is that we obtain a shrinking of Cooper pair size (that is of the order of $2-3 \mathrm{fm}$ ) at the nuclei surface compare to the center of the nucleus.
\end{abstract}

\section{Introduction}

Pairing correlations are of great interest in nuclear physics as they manifest in open-shell nuclei which constitute the majority of nuclei. These correlations have been included in microscopic models for a long time. They are commonly treated using the BCS or Hartree-Fock-Bogoliubov (HFB) theories. These methods suffer from defects that may be an obstacle to an accurate description of nuclear structure observables as, for example, particle number violation which hinders realistic description of pairing correlations in weak pairing regime. These difficulties can be obviated by employing particle number projection techniques [1]. However, such extensions require considerable additional numerical efforts. A comparison between exact and approximate treatments of the pairing interaction can be found in ref. [2] and references therein.

In view of this, an alternative approach has been proposed in ref. [3]. It consists in taking as trial wave function a linear combination of Slater determinants corresponding to multiple particle-hole excitations upon a Hartree-Fock (HF) state, which explicitly conserves particle numbers. Extensions of this pionnering work can be found in refs. [4] where the residual part of the hamiltonian is treated in a perturbative way, in the spirit of highly truncated shell model. In the present contribution, we discuss a variational and self-consistent derivation of such kind of method [5] using, for the first time, the same interaction for the mean field part and the residual part of the Hamiltonian, namely the D1S Gogny force [6]. Here, results are shown for ${ }^{68} \mathrm{Ni}$. Of course, such an approach supposes that a truncation in the number of configurations introduced in the trial wave function can be applied. Truncations related to the order of excitation of configurations or to the excitation energy of configurations have been studied in an exactly solvable model and compared to the exact solution, in the case of pairing Hamiltonian [7]. We should notice that the variational multiparticle-multihole (mpmh) configuration mixing method

${ }^{a}$ Presenting author, e-mail: nathalie.pillet@cea.fr is inspired with Multiconfiguration Hartree-Fock Method of atomic and molecular physics $[8,9]$.

Another interesting pairing feature is the spatial properties of this type of correlations. They have not been extensively studied in the past but, nevertheless, a certain number of investigations do exist both for nuclear matter and finite nuclei [10]. In our work, we use an HFB approach with the D1S Gogny force [6] to specify the Cooper pair spatial properties. Calculations have been made for spherical nuclei using a center of mass and relative coordinate representation [11].

In section 2 , we discuss the variational multiparticlemultihole configuration mixing method. Then, section 3 is devoted to pairing spatial properties.

\section{Variational multiparticle-multihole configuration mixing method}

We recall briefly the variational mpmh configuration mixing formalism. A detailed discussion can be found in ref. [5].

In the variational mpmh configuration mixing method, the trial wave function is taken as a superposition, truncated to finite order, of Slater determinants corresponding to mpmh excitations upon a HF-type ground state $\left|\phi_{\pi} \phi_{v}\right\rangle$

$$
|\Psi\rangle=A_{\pi v}^{0 p 0 h}\left|\phi_{\pi} \phi_{\nu}\right\rangle+\sum_{\alpha_{\pi} \alpha_{\nu}} A_{\alpha_{\pi} \alpha_{\nu}}^{1 p 1 h}\left|\phi_{\alpha_{\pi}} \phi_{\alpha_{\nu}}\right\rangle+\sum_{\alpha_{\pi} \alpha_{\nu}} A_{\alpha_{\pi} \alpha_{\nu}}^{2 p 2 h}\left|\phi_{\alpha_{\pi}} \phi_{\alpha_{\nu}}\right\rangle+\cdots
$$

The $A_{\alpha_{\pi} \alpha_{v}}^{m p m h}$ are mixing coefficients that depend on proton $(\pi)$ and neutron $(v)$ indices. The $\phi_{\alpha_{\pi}}, \phi_{\alpha_{v}}$ are Slater determinants of proton and neutron orbitals with a given order of combined excitation $\left(\alpha_{\pi}, \alpha_{v}\right) 1 \mathrm{p} 1 \mathrm{~h}, 2 \mathrm{p} 2 \mathrm{~h} . .$. In the spirit of the mean field approaches, a priori, no inert core is assumed in this method. Let $\hat{H}$ be the nuclear effective Hamiltonian

$$
\hat{H}=\hat{K}+\hat{V}[\rho]
$$

where $\hat{K}$ is the kinetic energy and $\hat{V}[\rho]$ an effective twobody density-dependent interaction. The one-body density $\rho$ is defined as: $\rho=\langle\Psi|\hat{\rho}| \Psi\rangle$. 
As the Slater determinants included in equation (1) span in a restricted part of the Hilbert space, the wave function (1) depends on two sets of quantities:

- the mixing coefficients $\left\{A_{\alpha_{\pi} \alpha_{v}}^{m p m h}\right\}$,

- the single-particle (sp) states entering the Slater determinant $\phi_{\alpha}$.

These quantities can be determined from a variational principle, that is from minimization of the functional

$$
\mathcal{F}[\rho]=E[\rho]-\lambda\langle\Psi \mid \Psi\rangle
$$

where $E[\rho]=\langle\Psi|\hat{H}[\rho]| \Psi\rangle$ is the total energy and $\lambda$ is a Lagrange parameter that ensures wave function norm conservation.

The minimization of the functional with respect to the mixing coefficients and the sp states leads respectively to two equations:

- a secular equation which is equivalent to a diagonalization problem in the multiconfiguration space, that gives the mixing coefficients,

- an inhomogeneous HF-type equation whose second member depends on the two-body correlation matrix $\sigma$, that provides sp states.

The full solution of the variational mpmh configuration mixing is accomplished through a self-consistent (SC) procedure where the two previous sets of equations can be solved simultaneously.

In this work, we have applied the variational mpmh configuration mixing method in the particular case of a trial wave function that includes only configurations corresponding to pair excitations of proton or neutron type, in order to describe pairing correlations. In our calculation, $\mathrm{sp}$ states used to build Slater determinants are developped on axially deformed harmonic oscillator basis. We have done our calculations with 11 shells that correspond to 286 single particle levels (doubly degenerated).

The correlation energy is defined as the difference between the total energy associated with $|\Psi\rangle$ and the total energy associated with the HF solution

$$
E_{c o r}=E[\rho]-E_{H F} .
$$

Figure 1 displays the evolution of the correlation energy for ${ }^{68} \mathrm{Ni}$ as a function of the number of neutron sp levels for a fixed number of proton configurations. All the proton configurations corresponding to $286 \mathrm{sp}$ levels have been included. Results are shown for wave functions including configurations up to one pair $(2 \mathrm{p} 2 \mathrm{~h})$, two pairs $(4 \mathrm{p} 4 \mathrm{~h})$. Contributions associated to three pair excitations are negligible in the case of ${ }^{68} \mathrm{Ni}$. Results concerning BCS approximation are also shown. The total correlation energy obtained in the mpmh configuration mixing approach is around $3.8 \mathrm{MeV}$. A calculation including only configurations associated to one pair excitation gives around $3.4 \mathrm{MeV}$. This means that adding configurations corresponding to two pair excitations corresponds to an energy gain of $400 \mathrm{keV}$. Correlations are dominated by one excited pair component that are built with sp levels close to the Fermi level. The slow convergence of the correlation energy can be

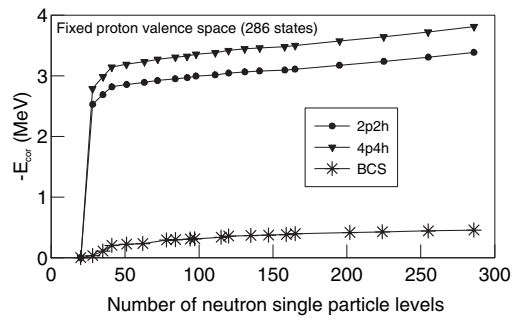

Fig. 1. Evolution of the mpmh correlation energy with respect to neutron valence space for ${ }^{68} \mathrm{Ni}$.

understood from the fact that the Gogny force involves sp levels up to energies of the order of $100 \mathrm{MeV}$ when pairing is strong. Therefore, a sufficient number of sp levels must be included. The comparison with BCS calculation shows that mpmh configuration mixing method is able to restore many correlations. In the case of ${ }^{68} \mathrm{Ni}$, the difference between the two approaches is of the order of $3.4 \mathrm{MeV}$.

If we look to the structure of the correlated wave function for ${ }^{68} \mathrm{Ni}$, we see that the HF component corresponds to $84 \%$ and the summed one excited pair component to $14.5 \%$. Two excited pair component contributes only to $1.5 \%$.

\section{Generic strong coupling behaviour of Cooper pairs in the surface of superfluid nuclei}

In the HFB approximation, the two-body correlation function is defined as

$$
\begin{aligned}
& \rho\left(\boldsymbol{r}_{1} s_{1}, \boldsymbol{r}_{2}-s_{2}, \boldsymbol{r}_{1} s_{1}, \boldsymbol{r}_{2}-s_{2}\right) \\
&=\left\langle\psi^{+}\left(\boldsymbol{r}_{1}, s_{1}\right) \psi^{+}\left(\boldsymbol{r}_{2},-s_{2}\right) \psi\left(\boldsymbol{r}_{2},-s_{2}\right) \psi\left(\boldsymbol{r}_{1}, s_{1}\right)\right\rangle \\
&= \rho\left(\boldsymbol{r}_{1} s_{1}, \boldsymbol{r}_{1} s_{1}\right) \rho\left(\boldsymbol{r}_{2}-s_{2}, \boldsymbol{r}_{2}-s_{2}\right) \\
&-\rho\left(\boldsymbol{r}_{1} s_{1}, \boldsymbol{r}_{2}-s_{2}\right) \rho\left(\boldsymbol{r}_{2} s_{2}, \boldsymbol{r}_{1} s_{1}\right)+\left|\kappa\left(\boldsymbol{r}_{1} s_{1}, \boldsymbol{r}_{2} s_{2}\right)\right|^{2} .
\end{aligned}
$$

In equation (5), $\langle\ldots\rangle=\langle H F B|\ldots| H F B\rangle, \psi^{+}$and $\psi$ are creation and annihilation operators, $\rho\left(\boldsymbol{r}_{1} s_{1}, \boldsymbol{r}_{2} s_{2}\right)=\left\langle\psi^{+}\left(\begin{array}{ll}\boldsymbol{r}_{2} & s_{2}\end{array}\right) \psi\right.$ $\left.\left(\begin{array}{ll}\boldsymbol{r}_{1} & s_{1}\end{array}\right)\right\rangle$ is the single particle density matrix and $\kappa\left(\boldsymbol{r}_{1} s_{1}\right.$, $\left.\boldsymbol{r}_{2} s_{2}\right)=\left\langle\psi\left(\boldsymbol{r}_{2}-s_{2}\right) \psi\left(\boldsymbol{r}_{1} s_{1}\right)\right\rangle$ is the pairing tensor in $r$-space. The first two terms on the r.h.s. of equation (5) represent the antisymmetrised mean field factorisation into products of single particle density matrices which also survive in the pure Hartree-Fock limit. It is the trivial and uncorrelated part of the density matrix. The genuine two-body correlations, of interest here, are contained in $|\kappa|^{2}$

$$
\kappa\left(\boldsymbol{r}_{1} s_{1} 1, \boldsymbol{r}_{2} s_{2}\right)=\left\langle\psi\left(\boldsymbol{r}_{2}-s_{2}\right) \psi\left(\boldsymbol{r}_{1} s_{1}\right)\right\rangle .
$$

We analyse the localisation properties of pairing tensor calculated in the HFB approximation and using the D1S Gogny force, for even-even muclei ground states $(\mathbf{J}=0)$. The HFB equations are solved in the harmonic oscillator basis. We have restricted the present study to spherical nuclei. In this case the pairing tensor corresponding to the total spin $S=0$ reads

$$
\begin{aligned}
\kappa\left(\boldsymbol{r}_{1}, \boldsymbol{r}_{2}\right) & =\sum_{s_{1}, s_{2}}\left\langle\psi\left(\boldsymbol{r}_{2}-s_{2}\right) \psi\left(\boldsymbol{r}_{1} s_{1}\right)\right\rangle \\
& =\frac{1}{4 \pi} \sum_{n, n^{\prime} l j} \frac{2 j+1}{2} \kappa_{n^{\prime}, n}^{l j} u_{n^{\prime} l}\left(r_{2}\right) u_{n l}\left(r_{1}\right)(-)^{l} P_{l}\left(\cos \theta_{21}\right)
\end{aligned}
$$




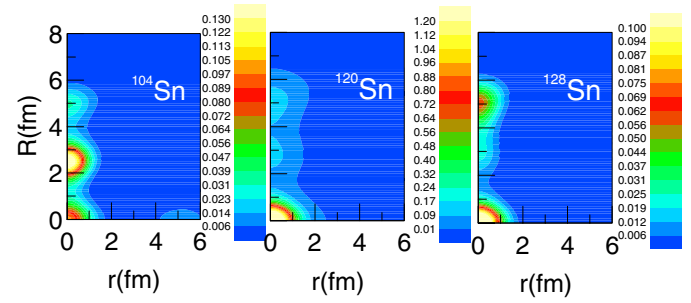

Fig. 2. $|\kappa(R, r)|^{2}$ calculated with HFB-D1S for ${ }^{104} \mathrm{Sn},{ }^{120} \mathrm{Sn},{ }^{128} \mathrm{Sn}$. Scale has been multiplied by a factor of $10^{6}$.

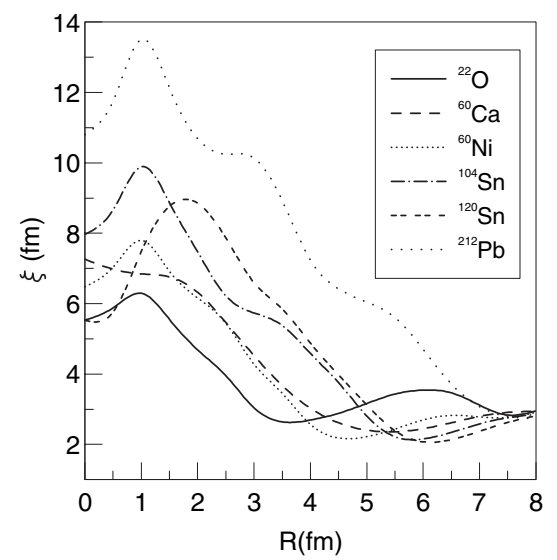

Fig. 3. Cooper pair size $\xi(R)$ for ${ }^{22} \mathrm{O},{ }^{60} \mathrm{Ca},{ }^{60} \mathrm{Ni},{ }^{104} \mathrm{Sn},{ }^{120} \mathrm{Sn},{ }^{212} \mathrm{~Pb}$.

where $u_{n l}(r)$ are the radial wave functions of the harmonic oscillator and $\kappa_{n^{\prime} n}^{l j}$ is the matrix of the pairing tensor for a given orbital and total angular momenta $l j$. As defined here, the latter has an intrinsic parity $(-)^{l}$.

The pairing tensor can be expressed in terms of relative and center of mass (CM) coordinates, i.e., $\boldsymbol{r}=\boldsymbol{r}_{1}-\boldsymbol{r}_{2}$ and $\boldsymbol{R}=\left(\boldsymbol{r}_{1}+\boldsymbol{r}_{2}\right) / 2$, using the Brody-Moshinski transformation. One thus gets

$$
\begin{aligned}
& \kappa(\boldsymbol{R}, \boldsymbol{r})=\frac{1}{4 \pi} \sum_{n, n^{\prime}, l_{1} j_{1}}\left(2 j_{1}+1\right) \kappa_{n_{2}, n_{1}}^{l_{1} j_{1}} \\
& \times \sum_{n N l}(-)^{l}\left(\frac{2 l+1}{2 l_{1}}\right)^{1 / 2} u_{n l}(r / \sqrt{2}) u_{N l}(\sqrt{2} R) \\
& \times P_{l}(\cos \theta)\left\langle n l N l ; 0 \mid n_{1} l_{1} n_{2} l_{1} ; 0\right\rangle
\end{aligned}
$$

where $\left\langle n l N l ; 0 \mid n_{1} l_{1} n_{2} l_{1} ; 0\right\rangle$ is the Brody-Moshinski bracket. In figure 2, we have represented $|\kappa(R, r)|^{2}$ for three tin isotopes. One can notice that for all tin isotopes the Cooper pairs have very small extension in r-direction throughout the nuclear radius. However, the distribution in $R$ is rather different in the three isotopes. The difference comes from the localisation properties of the single-particle shells which are closest to the chemical potential. Thus, the pronounced concentration of $|\kappa(R, r)|^{2}$ around $\mathrm{R}=5 \mathrm{fm}$ in ${ }^{128} \mathrm{Sn}$ is due to the surface localisation of the single-particle wave function $1 h_{11 / 2}$, which becomes much closer to the chemical potential in this isotope compared to lighter ones. One can also notice that in ${ }^{120,128} \mathrm{Sn}$ the pair probability has a sizeable value for small values of $R$, which comes mainly from the contribution of the state $3 s_{1 / 2}$ to pairing correlations.

The size of a Cooper pair is charaterized by its root mean square (rms) radius. Thus, for a fixed center of mass coordinate, the rms radius is:

$$
\zeta(R)=\frac{\left(\int r^{4} \kappa^{2}(r, R, \theta) d r \sin \theta d \theta\right)^{1 / 2}}{\left(\int r^{2} \kappa^{2}(r, R, \theta) d r \sin \theta d \theta\right)^{1 / 2}} .
$$

In figure 3, we have represented the rms radius for various nuclei. One sees well defined and pronounced minima at $\xi \sim 2-3 f m$ for $\mathrm{R}$ of the order of the surface radius, for all nuclei. This shows a shrinking of the size of Cooper pairs from the center to the nuclear surface. This seems to indicate that, in the nucleus, Cooper pairs are in a strong coupling regime.

\section{References}

1. J.A. Sheikh, P. Ring, Nucl. Phys. A 665, 71 (2000); M. Anguino, J.L. Egido, L.M. Robledo, Nucl. Phys. A 696, 467 (2001); M. Anguino, J.L. Egido, L.M. Robledo, Phys. Lett. B 545, 62 (2002).

2. J. Dukelsky, G.G. Dussel, J.G. Hirsch, P. Schuck, Nucl. Phys. A 714, 63 (2003).

3. N. Pillet, P. Quentin, J. Libert, Nucl. Phys. A 697, 141 (2002).

4. T.L. Ha, Ph.D. thesis, Université Bordeaux 1 (unpublished); P. Quentin, H. Laftchiev, D. Samsoen, I.N. Mikhailov, J. Libert, Nucl. Phys. A 734, 477 (2004); K. Sieja, T.L. Ha, P. Quentin, A. Baran, Int. J. Mod. Phys. E 16, 289 (2007); L. Bonneau, P. Quentin, K. Sieja (submitted to publication, arXiv:nuclth/0703102).

5. N. Pillet, J.-F. Berger, E. Caurier, H. Goutte (to be submitted); N. Pillet, J.-F. Berger, E. Caurier, M. Girod, Int. J. Mod. Phys. E 15, 464 (2006).

6. J. Dechargé, D. Gogny, Phys. Rev. C 21, 1568 (1980); J.-F. Berger, M. Girod, D. Gogny, Comp. Phys. Comm. 63, 365 (1991).

7. N. Pillet, N. Sandulescu, Nguyen Van Giai, J.-F. Berger, Phys. Rev. C 71, 044306 (2005).

8. C. Froese-Fischer, Comp. Phys. Comm. 1, 151 (1969); J.C. Morrison, C. Froese-Fischer, Phys. Rev. A 35, 2429 (1987).

9. D.L. Yeager, P. Jorgensen, J. Chem. Phys. 71, 2 (1979); H.J. Werner, W. Meyer, J. Chem. Phys. 74, 10 (1981).

10. R.H. Ibarra, N. Austern, M. Vallieres, D.H. Feng, Nucl. Phys. A 288, 397 (1977); F. Catara, A. Insolia, E. Maglione, A. Vitturi, Phys. Rev. C 29, 1091 (1984); L. Ferreira, R. Liotta, C.H. Dasso, R.A. Broglia, A. Winther, Nucl. Phys. A 426, 276 (1984); G.F. Bertsch, H. Esbensen, Ann. Phys. 209, 327 (1991); H. Esbensen, G.F. Bertsch, K. Hencken, Phys. Rev. C 56, 3054 (1999); K. Hagino, H. Sagawa, J. Carbonell, P. Schuck (submitted to publication, arXiv:nucl-th/0611064v1); M.A. Tischler, A. Tonina, G.G. Dussel, Phys. Rev. C 58, 2591 (1998); F. Barranco, R.A. Broglia, H. Esbensen, E.Vigezzi, Phys. Rev. C 58, 1257 (1998); M. Matsuo, K. Mizuyama, Y. Serizawa, Phys. Rev. C 71, 064326 (2005); M. Farine, P. Schuck, Phys. Lett. B 459, 444 (1999); M. Baldo, U. Lombardo, E. Saperstein, M. Zverev, Phys. Lett. B 459, 437 (1999).

11. N. Pillet, N. Sandulescu, P. Schuck (submitted to publication, arXiv:nucl-th/0701086). 